International Journal of Pure and Applied Mathematics

Volume 86 No. 2 2013, 411-424

ISSN: 1311-8080 (printed version); ISSN: 1314-3395 (on-line version)

url: http://www.ijpam.eu

doi: http://dx.doi.org/10.12732/ijpam.v86i2.15

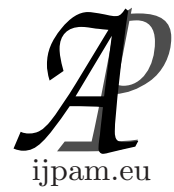

\title{
T-ROUGH IDEALS IN TERNARY SEMIGROUPS
}

\author{
Moin A. Ansari ${ }^{1 \mathbb{S}}$, Naveed Yaqoob ${ }^{2}$ \\ ${ }^{1}$ Department of Mathematics \\ Faculty of Science \\ Post Box 2097, Jazan University \\ Jazan, KINGDOM OF SAUDI ARABIA \\ ${ }^{2}$ Department of Mathematics \\ Quaid-i-Azam University \\ Islamabad, PAKISTAN
}

\begin{abstract}
The purpose of this paper is to introduce and discuss the concept of T-rough ternary subsemigroups, T-rough ideals, T-rough bi-ideals and T-rough interior ideals in ternary semigroups.
\end{abstract}

AMS Subject Classification: 20N10

Key Words: ternary semigroups, rough sets, T-rough sets, T-rough ideals

\section{Introduction}

The notion of a rough set was proposed by Pawlak [1] in 1982. Since then the subject has been investigated in many papers. There are many mathematicians who applied the rough set theory to algebraic structures, for instance, Biswas and Nanda [2], Xiao and Zhang [3], Petchkhaew and Chinram [4], Kuroki [5], Shabir and Rehman [6], Yaqoob et al. [7-16], Davvaz [17, 18, 19], Kazancı et al. [20] and Leoreanu [21].

The majority studies on rough sets for algebraic structures such as semigroups, groups, rings and modules have been concentrated on a congruence relation. However, the congruence relation restricts the application of the generalized rough set model for algebraic sets. To solve this problem, Davvaz [22]

Received: March 25, 2013

(c) 2013 Academic Publications, Ltd.

${ }^{\mathbb{S}}$ Correspondence author 
introduced the concept of set-valued homomorphism in groups. Xiao [23], studied the properties of T-roughness in semigroups. Hosseini et al. [24, 25], applied T-rough set theory to semigroups and modules.

In this paper, the concept of set-valued homomorphism of ternary semigroups is defined. We constructed the generalized rough approximation operators in ternary semigroups by means of set-valued mapping and the properties of them are studied.

\section{Preliminaries and Basic Definitions}

In this section, we give some basic notions and results about generalized rough sets and ternary semigroups.

Definition 2.1. [23] Let $U$ and $W$ be two non-empty universes. Let $T$ be a set-valued mapping given by $T: U \rightarrow P(W)$. Then the triple $(U, W, T)$ is referred to as a generalized approximation space or generalized rough set. Any set-valued function from $U$ to $P(W)$ defines a binary relation from $U$ to $W$ by setting $R_{T}=\{(x, y) \mid y \in T(x)\}$. Obviously, if $R$ is an arbitrary relation from $U$ to $W$, then it can be defined as a set-valued mapping $T_{R}: U \rightarrow P(W)$ by $T_{R}(x)=\{y \in W \mid(x, y) \in R\}$ where $x \in U$. For any set $A \subseteq W$, the lower and upper approximations $\underline{A p r}_{T}(A)$ and $\overline{A p r}_{T}(A)$ are defined by

$$
\begin{aligned}
& \overline{A p r}_{T}(A)=\{x \in U \mid T(x) \subseteq A\} \\
& \overline{A p r}_{T}(A)=\{x \in U \mid T(x) \cap A \neq \emptyset\} .
\end{aligned}
$$

The pair $\left(\underline{A p r}_{T}(A), \overline{A p r}_{T}(A)\right)$ is referred to as a generalized rough set, and $\underline{A p r}_{T}$ and $\overline{A p r}_{T}$ are referred to as lower and upper generalized approximation operators, respectively.

If a subset $A \subseteq W$ satisfies that $\operatorname{Apr}_{T}(A)=\overline{A p r}_{T}(A)$, then $A$ is called a definable set of $(U, W, T)$. We denote all the definable sets of $(U, W, T)$ by $\operatorname{Def}(T)$.

Theorem 2.2. [23] Let $(U, W, T)$ be a generalized approximation space, its lower and upper approximation operators satisfy the following properties: 
For all $A, B \in P(W)$,

$$
\begin{aligned}
& \text { (L1) } \operatorname{Apr}_{T}(A)=\left(\overline{A p r}_{T}\left(A^{c}\right)\right)^{c} \text {, } \\
& \text { (L2) } \overline{A p r}_{T}(W)=U \text {, } \\
& \text { (L3) } \underline{A p r}_{T}(A \cap B)=\underline{A p r}_{T}(A) \cap \underline{A p r}_{T}(B) \text {, } \\
& (L 4) A \subseteq B \Rightarrow \underline{A p r}_{T}(A) \subseteq \underline{A p r}_{T}(B), \\
& \text { (L5) } \underline{A p r}_{T}(A \cup B) \supseteq \underline{A p r}_{T}(A) \cup \underline{A p r}_{T}(B) \text {, } \\
& \text { (U1) } \overline{\operatorname{Apr}}_{T}(A)=\left(\underline{A p r}_{T}\left(A^{c}\right)\right)^{c} \text {, } \\
& \text { (U2) } \overline{A p r}_{T}(\emptyset)=\emptyset \text {, } \\
& \text { (U3) } \overline{A p r}_{T}(A \cup B)=\overline{A p r}_{T}(A) \\
& \cup \overline{A p r}_{T}(B) \text {, } \\
& \text { (U4) } A \subseteq B \Rightarrow \\
& \overline{A p r}_{T}(A) \subseteq \overline{A p r}_{T}(B), \\
& \text { (U5) } \overline{A p r}_{T}(A \cap B) \\
& \subseteq \overline{A p r}_{T}(A) \cap \overline{A p r}_{T}(B) .
\end{aligned}
$$

where $A^{c}$ is the complement of the set $A$.

Definition 2.3. A ternary semigroup is an algebraic structure $(S, f)$ such that $S$ is a non-empty set and $f: S^{3} \rightarrow S$ is a ternary operation satisfying the following associative law:

$$
f(f(a, b, c), d, e)=f(a, f(b, c, d), e)=f(a, b, f(c, d, e)) .
$$

For simplicity we write $f(a, b, c)$ as $a b c$.

Definition 2.4. A non-empty subset $T$ of a ternary semigroup $S$ is said to be a ternary subsemigroup of $S$ if $T T T=T^{3} \subseteq T$, that is $a b c \in T$ for all $a, b, c \in T$.

Definition 2.5. By a left (right, lateral (middle)) ideal of a ternary semigroup $S$ we mean a non-empty subset $A$ of $S$ such that $S S A \subseteq A(A S S \subseteq$ $A, S A S \subseteq A$ ). By a two sided ideal, we mean a subset of $S$ which is both a left and a right ideal of $S$. If a non-empty subset of $S$ is a left, right and lateral ideal of $S$, then it is called an ideal of $S$.

Definition 2.6. An ideal $A$ of a semigroup $S$ is a prime ideal of $S$ such that $x y z \in A$ for some $x, y, z \in S$ implies $x \in A$ or $y \in A$ or $z \in A$.

Definition 2.7. A non-empty subset $A$ of a ternary semigroup $S$ is called a bi-ideal of $S$ if $A A A \subseteq A$ and $A S A S A \subseteq A$.

Definition 2.8. A bi-ideal $A$ of a ternary semigroup $S$ is said to be a prime bi-ideal of $S$, if for $x, y, z \in S, x a y b z \in A$ implies $x \in A$ or $y \in A$ or $z \in A$ for all $a, b \in S$.

Definition 2.9. A non-empty subset $A$ of $S$ is called an interior ideal of $S$ if $S S A S S \subseteq A$.

Definition 2.10. A non-empty subset $A$ of $S$ is called a quasi-ideal of $S$ if $A S S \cap S A S \cap S S A \subseteq A$ and $A S S \cap S S A S S \cap S S A \subseteq A$. 
Definition 2.11. Let $\rho$ be a congruence on ternary semigroup $S$, that is, $\rho$ is an equivalence relation on $S$ such that

$$
(a, b) \in \rho \text { implies }(x y a, x y b) \in \rho,(x a y, x b y) \in \rho \text { and }(a x y, b x y) \in \rho
$$

for all $a, b, x, y \in S$.

We denote by $[a]_{\rho}$ the $\rho$-congruence class containing the element $a \in S$. It is obvious that $[a]_{\rho}[b]_{\rho}[c]_{\rho} \subseteq[a b c]_{\rho}$ for all $a, b, c \in S$. A congruence $\rho$ on $S$ is called complete if $[a]_{\rho}[b]_{\rho}[c]_{\rho}=[a b c]_{\rho}$ for all $a, b, c \in S$.

\section{T-rough Ideals in Ternary Semigroups}

In what follows, let $S$ denote a ternary semigroup unless otherwise specified. In this section, we will discuss some results on T-roughness in ternary semigroups.

Definition 3.1. Let $S_{1}$ and $S_{2}$ be two ternary semigroups and $B \subseteq S_{2}$. Let $T: S_{1} \rightarrow P\left(S_{2}\right)$ be a set-valued mapping where $P\left(S_{2}\right)$ denotes the set of all non-empty subsets of $S_{2}$. The generalized lower and upper approximations of $B$ are defined by

$$
\begin{aligned}
& \overline{A p r}_{T}(B)=\left\{x \in S_{1}: T(x) \subseteq B\right\} \\
& \overline{A p r}_{T}(B)=\left\{x \in S_{1}: T(x) \cap B \neq \emptyset\right\} .
\end{aligned}
$$

Definition 3.2. A set-valued homomorphism $T$ from a ternary semigroup $S_{1}$ to a ternary semigroup $S_{2}$ is a mapping from $S_{1}$ to $P\left(S_{2}\right)$ that preserves the group operation, that is, $T(a) T(b) T(c) \subseteq T(a b c)$ for all $a, b, c \in S_{1}$. T is called a strong set-valued homomorphism, if $T(a) T(b) T(c)=T(a b c)$ for all $a, b, c \in S_{1}$.

Example 3.3. Let $S_{1}=\{x, y, z\}$ and $S_{2}=\{a, b, c, d, e\}$ be two ternary semigroups with the following multiplication tables:

\begin{tabular}{c|ccc} 
& $x$ & $y$ & $z$ \\
\hline$x$ & $x$ & $x$ & $x$ \\
$y$ & $x$ & $y$ & $y$ \\
$z$ & $x$ & $z$ & $z$
\end{tabular}

\begin{tabular}{c|lllll} 
& $a$ & $b$ & $c$ & $d$ & $e$ \\
\hline$a$ & $d$ & $b$ & $b$ & $d$ & $b$ \\
$b$ & $b$ & $b$ & $b$ & $b$ & $b$ \\
$c$ & $b$ & $b$ & $c$ & $b$ & $c$ \\
$d$ & $d$ & $b$ & $b$ & $d$ & $b$ \\
$e$ & $b$ & $b$ & $c$ & $b$ & $c$
\end{tabular}

Assume $T(x)=\{a, d\}, T(y)=\{b, c\}$ and $T(z)=\{e\}$. Here $T$ is a set-valued homomorphism from $S_{1}$ to $S_{2}$. But $T$ is not a strong set-valued homomorphism from $S_{1}$ to $S_{2}$, because

$$
\{d\}=T(x) T(x) T(x) \neq T(x x x)=\{a, d\} .
$$


Theorem 3.4. Let $\theta$ be a (complete) congruence relation on a ternary semigroup $S$. Define $T_{\theta}: S \rightarrow P(S)$ by $T_{\theta}(x)=[x]_{\theta}$. Then $T_{\theta}$ is a (strong) set-valued homomorphism.

Theorem 3.5. Let $S_{1}$ and $S_{2}$ be two ternary semigroups and $T: S_{1} \rightarrow$ $P\left(S_{2}\right)$ be a set-valued homomorphism. If $A, B, C$ are three non-empty subsets of $S_{2}$, then

$$
\overline{A p r}_{T}(A) \overline{A p r}_{T}(B) \overline{A p r}_{T}(C) \subseteq \overline{A p r}_{T}(A B C) .
$$

Proof. Assume that $t \in \overline{A p r}_{T}(A) \overline{A p r}_{T}(B) \overline{A p r}_{T}(C)$, there exist $x \in \overline{A p r}_{T}(A)$, $y \in \overline{A p r}_{T}(B)$ and $z \in \overline{A p r}_{T}(C)$ such that $t=x y z$. So there exist $a \in T(x) \cap A$, $b \in T(y) \cap B$ and $c \in T(z) \cap C$, then $a b c \in A B C$. Since $T$ is a set-valued homomorphism, we have

$$
a b c \in T(x) T(y) T(z) \subseteq T(x y z)=T(t) .
$$

So $a b c \in T(t) \cap A B C$, which implies $t \in T(A B C)$.

Theorem 3.6. Let $S_{1}$ and $S_{2}$ be two ternary semigroups and $T: S_{1} \rightarrow$ $P\left(S_{2}\right)$ be a strong set-valued homomorphism. If $A, B, C$ are three non-empty subsets of $S_{2}$, then

$$
\underline{A p r}_{T}(A) \underline{A p r}_{T}(B) \underline{A p r}_{T}(C) \subseteq \underline{A p r}_{T}(A B C) .
$$

Proof. Assume that $t \in \underline{A p r}_{T}(A) \underline{A p r}_{T}(B) \underline{A p r}_{T}(C)$, there exist $x \in \underline{A p r}_{T}(A)$, $y \in \underline{A p r}_{T}(B)$ and $z \in \underline{A p r}_{T} \overline{(C)}$ such that $t=\overline{x y z}$. Since $T$ is a strong set-valued homomorphism, we have

$$
T(t)=T(x y z)=T(x) T(y) T(z) \subseteq A B C .
$$

Thus $t \in T(A B C)$.

Let $T_{1}, T_{2}$ and $T_{3}$ be three set-valued mappings from $S_{1}$ to $P\left(S_{2}\right)$, we define

$$
\left(T_{1} \cap T_{2} \cap T_{3}\right)(x)=T_{1}(x) \cap T_{2}(x) \cap T_{3}(x) .
$$

Theorem 3.7. Let $T_{1}, T_{2}$ and $T_{3}$ be three set-valued mappings from $S_{1}$ to $\wp^{*}\left(S_{2}\right)$. If $A$ is a non-empty subset of $S_{2}$, then

$$
\begin{aligned}
& \text { (1) } \overline{A p r}_{T_{1} \cap T_{2} \cap T_{3}}(A) \subseteq \overline{A p r}_{T_{1}}(A) \cap \overline{A p r}_{T_{2}}(A) \cap \overline{A p r}_{T_{3}}(A) \\
& \text { (2) } \underline{A p r}_{T_{1} \cap T_{2} \cap T_{3}}(A) \supseteq \underline{A p r} \underline{T}_{1}(A) \cap \underline{A p r}_{T_{2}}(A) \cap \underline{A p r}_{T_{3}}(A) .
\end{aligned}
$$


Proof. (1) Assume that $x \in \overline{A p r}_{T_{1} \cap T_{2} \cap T_{3}}(A)$, there exists $y \in\left(T_{1} \cap T_{2} \cap\right.$ $\left.T_{3}\right)(x) \cap A$. So $y \in T_{1}(x) \cap A, y \in T_{2}(x) \cap A$, and $y \in T_{3}(x) \cap A$, which imply that $x \in \overline{A p r}_{T_{1}}(A) \cap \overline{A p r}_{T_{2}}(A) \cap \overline{A p r}_{T_{3}}(A)$.

(2) Assume that $x \in \underline{A p r}_{T_{1}}(A) \cap \underline{A p r}_{T_{2}}(A) \cap \underline{A p r}_{T_{3}}(A)$, we have $T_{1}(x) \subseteq A$, $T_{2}(x) \subseteq A$ and $T_{3}(x) \subseteq A$. So $T_{1}(x) \cap T_{2}(x) \cap T_{3}(x) \subseteq A$. Thus $\left(T_{1} \cap T_{2} \cap T_{3}\right)(x) \subseteq$ $A$, which implies that $x \in \underline{A p r}_{T_{1} \cap T_{2} \cap T_{3}}(A)$.

Theorem 3.8. Let $S_{1}$ and $S_{2}$ be two ternary semigroups and $T: S_{1} \rightarrow$ $P\left(S_{2}\right)$ be a set-valued homomorphism. If $A, B$ and $C$ are a right ideal, lateral ideal and a left ideal of $S_{2}$, respectively. Then

$$
\begin{aligned}
& \text { (1) } \overline{A p r}_{T}(A B C) \subseteq \overline{A p r}_{T}(A) \cap \overline{A p r}_{T}(B) \cap \overline{A p r}_{T}(C) \\
& \text { (2) } \underline{A p r}_{T}(A B C) \subseteq \underline{A p r}{ }_{T}(A) \cap \underline{A p r}_{T}(B) \cap \underline{A p r}_{T}(C) .
\end{aligned}
$$

Proof. (1) Since $A$ is a right ideal of $S_{2}$, so $A B C \subseteq A S_{2} S_{2} \subseteq A$. Since $B$ is a lateral ideal of $S_{2}$, so $A B C \subseteq S_{2} B S_{2} \subseteq B$, also $C$ is a left ideal of $S_{2}$, so $A B C \subseteq S_{2} S_{2} C \subseteq C$, thus $A B C \subseteq A \cap B \cap C$. Then by Theorem 2.2(U4) and Theorem 2.2(U5), we have

$$
\begin{aligned}
\overline{A p r}_{T}(A B C) & \subseteq \overline{A p r}_{T}(A \cap B \cap C) \\
& \subseteq \overline{A p r}_{T}(A) \cap \overline{A p r}_{T}(B) \cap \overline{A p r}_{T}(C) .
\end{aligned}
$$

(2) The proof is similar to (1).

Theorem 3.9. Let $S_{1}$ and $S_{2}$ be two ternary semigroups and $T: S_{1} \rightarrow$ $P\left(S_{2}\right)$ be a set-valued homomorphism.

(1) If $A$ is a ternary subsemigroup of $S_{2}$. Then $\overline{A p r}_{T}(A)$ is, if it is nonempty, a ternary subsemigroup of $S_{1}$.

(2) If $A$ is a left [right, lateral] ideal of $S_{2}$ and $T(x) \neq \emptyset$ for all $x \in S$. Then $\overline{A p r}_{T}(A)$ is, if it is non-empty, a left [right, lateral] ideal of $S_{1}$.

Proof. (1) Since $A$ is a ternary subsemigroup of $S_{2}$, we have $A A A \subseteq A$. By Theorem 2.2 and Theorem 3.5, we have

$$
\overline{A p r}_{T}(A) \overline{A p r}_{T}(A) \overline{A p r}_{T}(A) \subseteq \overline{A p r}_{T}(A A A) \subseteq \overline{A p r}_{T}(A) .
$$

So $\overline{A p r}_{T}(A)$ is a ternary subsemigroup of $S_{1}$.

(2) Since $A$ is a left ideal of $S_{2}$, we have $S_{2} S_{2} A \subseteq A$. So $\overline{A p r}_{T}\left(S_{2} S_{2} A\right) \subseteq$ $\overline{A p r}_{T}(A)$. Since $T(x) \neq \emptyset$ for all $x \in S$, we have $\overline{A p r}_{T}\left(S_{2}\right)=S_{1}$. So

$$
S_{1} S_{1} \overline{A p r}_{T}(A)=\overline{A p r}_{T}\left(S_{2}\right) \overline{A p r}_{T}\left(S_{2}\right) \overline{A p r}_{T}(A)
$$




$$
\subseteq \overline{A p r}_{T}\left(S_{2} S_{2} A\right) \subseteq \overline{A p r}_{T}(A) .
$$

This means that $\overline{A p r}_{T}(A)$ is, if it is non-empty, a left ideal of $\mathrm{S}$. The other cases can be seen in a similar way.

Theorem 3.10. Let $S_{1}$ and $S_{2}$ be two ternary semigroups and $T: S_{1} \rightarrow$ $P\left(S_{2}\right)$ be a strong set-valued homomorphism.

(1) If $A$ is a ternary subsemigroup of $S_{2}$. Then $\underline{A p r}_{T}(A)$ is, if it is nonempty, a ternary subsemigroup of $S_{1}$.

(2) If $A$ is a left [right, lateral] ideal of $S_{2}$. Then $\underline{A p r}_{T}(A)$ is, if it is nonempty a left [right, lateral] ideal of $S_{1}$.

Proof. (1) Since $A$ be a ternary subsemigroup of $S_{2}$, we have $A A A \subseteq A$. By Theorem 2.2 and Theorem 3.6, we have

$$
\underline{A p r}_{T}(A) \underline{A p r}_{T}(A) \underline{A p r}_{T}(A) \subseteq \underline{A p r}_{T}(A A A) \subseteq \underline{A p r}_{T}(A) .
$$

So $\underline{A p r}_{T}(A)$ is a ternary subsemigroup of $S_{1}$.

(2) Since $A$ is a left ideal of $S_{2}$, we have $S_{2} S_{2} A \subseteq A$. So $\underline{A p r}_{T}\left(S_{2} S_{2} A\right) \subseteq$ $\underline{A p r}_{T}(A)$, we have $\underline{A p r}_{T}\left(S_{2}\right)=S_{1}$. So

$$
\begin{aligned}
S_{1} S_{1} \underline{A p r}_{T}(A) & =\underline{A p r}_{T}\left(S_{2}\right) \underline{A p r}_{T}\left(S_{2}\right) \underline{A p r}_{T}(A) \\
& \subseteq \underline{A p r}_{T}\left(S_{2} S_{2} A\right) \subseteq \underline{A p r}_{T}(A) .
\end{aligned}
$$

This means that $\underline{A p r}_{T}(A)$ is, if it is non-empty a left ideal of $S_{1}$.

The other cases can be seen in a similar way.

Theorem 3.11. Let $S_{1}$ and $S_{2}$ be two ternary semigroups and $T: S_{1} \rightarrow$ $P\left(S_{2}\right)$ be a strong set-valued homomorphism. If $A$ is a prime ideal of $S_{2}$, then

(1) $\overline{A p r}_{T}(A)$ is, if it is non-empty, a prime ideal of $S_{1}$,

(2) $\operatorname{Apr}_{T}(A)$ is, if it is non-empty, a prime ideal of $S_{1}$.

Proof. By Theorem 3.9(2) and Theorem 3.10(2), we know $\overline{A p r}_{T}(A)$ and $\underline{A p r}_{T}(A)$ are ideals of $S_{1}$.

(1) Assume that $x y z \in \overline{A p r}_{T}(A)$, then $T(x y z) \cap A \neq \emptyset$. Since $T$ is a strong set-valued homomorphism, we have $T(x y z)=T(x) T(y) T(z)$. So there exist $u \in T(x), v \in T(y)$ and $w \in T(z)$ such that $u v w \in A$. Since $A$ is a prime ideal of $S_{2}$, we have $u \in A$ or $v \in A$ or $w \in A$. So $x \in \overline{A p r}_{T}(A)$ or $y \in \overline{A p r}_{T}(A)$ or $z \in \overline{A p r}_{T}(A)$. Therefore, $\overline{A p r}_{T}(A)$ is, if it is non-empty, a prime ideal of $S_{1}$.

(2) We suppose that $\operatorname{Apr}_{T}(A)$ is not a prime ideal of $S_{1}$, then there exist $x, y, z \in S_{1}$ such that $x y z \in \underline{A p r}_{T}(A)$ but $x \notin \underline{A p r}_{T}(A), y \notin \underline{A p r}_{T}(A)$ and 
$z \notin \underline{A p r}_{T}(A)$. So there exist $a \in T(x), b \in T(y)$ and $c \in T(z)$ but $a, b, c \notin A$. Thus

$$
a b c \in T(x) T(y) T(z)=T(x y z) \subseteq A .
$$

Since $A$ is a prime ideal of $S_{2}$, we have $a \in A$ or $b \in A$ or $c \in A$. It contradicts the supposition. This means that $\operatorname{Apr}_{T}(A)$ is, if it is non-empty, a prime ideal of $S_{1}$.

Theorem 3.12. Let $S_{1}$ and $S_{2}$ be two ternary semigroups and $T: S_{1} \rightarrow$ $P\left(S_{2}\right)$ be a set-valued homomorphism. If $A$ is a bi-ideal of $S_{2}$ and $T(x) \neq \emptyset$ for all $x \in S$, then $\overline{A p r}_{T}(A)$ is, if it is non-empty, a bi-ideal of $S_{1}$.

Proof. Since $A$ is a bi-ideal of $S_{2}$, we have $A S_{2} A S_{2} A \subseteq A$. Since $T(x) \neq \emptyset$ for all $x \in S$, we have $\overline{A p r}_{T}\left(S_{2}\right)=S_{1}$. By Theorem 3.5, we have

$$
\begin{aligned}
\overline{A p r}_{T}(A) S_{1} \overline{A p r}_{T}(A) S_{1} \overline{A p r}_{T}(A) & \\
= & \overline{A p r}_{T}(A) \overline{A p r}_{T}\left(S_{2}\right) \overline{A p r}_{T}(A) \overline{A p r}_{T}\left(S_{2}\right) \overline{A p r}_{T}(A) \\
& \subseteq \overline{A p r}_{T}\left(A S_{2} A S_{2} A\right) \subseteq \overline{A p r}_{T}(A) .
\end{aligned}
$$

By this and Theorem 3.9(1), $\overline{A p r}_{T}(A)$ is, if it is non-empty, a bi-ideal of $S_{1}$.

Theorem 3.13. Let $S_{1}$ and $S_{2}$ be two ternary semigroups and $T: S_{1} \rightarrow$ $P\left(S_{2}\right)$ be a strong set-valued homomorphism. If $A$ is a bi-ideal of $S_{2}$, then $\operatorname{Apr}_{T}(A)$ is, if it is non-empty, a bi-ideal of $S_{1}$.

Proof. Since $A$ is a bi-ideal of $S_{2}$, we have $A S_{2} A S_{2} A \subseteq A$. So $T\left(A S_{2} A S_{2} A\right) \subseteq$ $T(A)$, we have $\overline{A p r}_{T}\left(S_{2}\right)=S_{1}$. By Theorem 3.6, we have

$$
\begin{aligned}
& \underline{A p r}_{T}(A) S_{1} \underline{A p r}_{T}(A) S_{1} \underline{A p r}_{T}(A) \\
& =\underline{A p r}_{T}(A) \underline{A p r}_{T}\left(S_{2}\right) \underline{A p r}_{T}(A) \underline{A p r} \underline{T}_{T}\left(S_{2}\right) \underline{A p r}_{T}(A) \\
& \subseteq \underline{A p r}_{T}\left(A S_{2} A S_{2} A\right) \subseteq \underline{A p r} T(A) .
\end{aligned}
$$

By this and Theorem 3.10(1), $\underline{A p r}_{T}(A)$ is, if it is non-empty a bi-ideal of $S_{1}$.

Theorem 3.14. Let $S_{1}$ and $S_{2}$ be two ternary semigroups and $T: S_{1} \rightarrow$ $P\left(S_{2}\right)$ be a strong set-valued homomorphism. If $A$ is a prime bi-ideal of $S_{2}$, then

(1) $\overline{A p r}_{T}(A)$ is, if it is non-empty, a prime bi-ideal of $S_{1}$,

(2) $\operatorname{Apr}_{T}(A)$ is, if it is non-empty, a prime bi-ideal of $S_{1}$. 
Proof. By Theorem 3.12 and 3.13, we know $\overline{A p r}_{T}(A)$ and $\underline{A p r} \underline{T}_{T}(A)$ are bi-ideals of $S_{1}$.

(1) Assume that $x a y b z \in \overline{A p r}_{T}(A)$, then $T(x a y b z) \cap A \neq \emptyset$. Since $T$ is a strong set-valued homomorphism, we have

$$
T(x a y b z)=T(x) T(a) T(y) T(b) T(z) .
$$

So there exist $s \in T(x), t \in T(a), u \in T(y), v \in T(b)$ and $w \in T(z)$ such that stuvw $\in A$. Since $A$ is a prime bi-ideal of $S_{2}$, we have $s \in A$ or $u \in A$ or $w \in A$. So $x \in \overline{A p r}_{T}(A)$ or $y \in \overline{A p r}_{T}(A)$ or $z \in \overline{A p r}_{T}(A)$. Therefore, $\overline{A p r}_{T}(A)$ is, if it is non-empty, a prime bi-ideal of $S_{1}$.

(2) We suppose that $\operatorname{Apr}_{T}(A)$ is not a prime bi-ideal of $S_{1}$, then there exist $x, a, y, b, z \in S_{1}$ such that $x a y b z \in \underline{A p r}_{T}(A)$ but $x \notin \underline{A p r}_{T}(A), y \notin \underline{A p r}_{T}(A)$ and $z \notin \underline{A p r}_{T}(A)$. So there exist $s \overline{\in T}^{T}(x), t \in T(a), \overline{u \in T}^{T}(y), v \in \overline{T(b)}^{T}$ and $w \in T(z)$ but $s, t, u, v, w \notin A$. Thus

$$
\text { stuvw } \in T(x) T(a) T(y) T(b) T(z)=T(x a y b z) \subseteq A .
$$

Since $A$ is a prime bi-ideal of $S_{2}$, we have $s \in A$ or $u \in A$ or $w \in A$. It contradicts the supposition. This means that $\operatorname{Apr}_{T}(A)$ is, if it is non-empty, a prime bi-ideal of $S_{1}$.

Theorem 3.15. Let $S_{1}$ and $S_{2}$ be two ternary semigroups and $T: S_{1} \rightarrow$ $P\left(S_{2}\right)$ be a set-valued homomorphism. If $A$ is an interior ideal of $S_{2}$ and $T(x) \neq \emptyset$ for all $x \in S$, then $\overline{A p r}_{T}(A)$ is, if it is non-empty, an interior ideal of $S_{1}$.

Proof. The proof is similar to the proof of Theorem 3.12.

Theorem 3.16. Let $S_{1}$ and $S_{2}$ be two ternary semigroups and $T: S_{1} \rightarrow$ $P\left(S_{2}\right)$ be a strong set-valued homomorphism. If $A$ is an interior ideal of $S_{2}$, then $\underline{A p r}_{T}(A)$ is, if it is non-empty, an interior ideal of $S_{1}$.

Proof. The proof is similar to the proof of Theorem 3.13.

Theorem 3.17. Let $S_{1}$ and $S_{2}$ be two ternary semigroups and $T: S_{1} \rightarrow$ $P\left(S_{2}\right)$ be a strong set-valued homomorphism. If $A$ is a quasi-ideal of $S_{2}$, then $\underline{A p r}_{T}(A)$ is, if it is non-empty, a quasi-ideal of $S_{1}$.

Proof. Let $A$ be a quasi-ideal of $S_{2}$, we have $A S_{2} S_{2} \cap S_{2} A S_{2} \cap S_{2} S_{2} A \subseteq A$ and $A S_{2} S_{2} \cap S_{2} S_{2} A S_{2} S_{2} \cap S_{2} S_{2} A \subseteq A$. So $T\left(A S_{2} S_{2} \cap S_{2} A S_{2} \cap S_{2} S_{2} A\right) \subseteq$ 
$T(A)$ and $T\left(A S_{2} S_{2} \cap S_{2} S_{2} A S_{2} S_{2} \cap S_{2} S_{2} A\right) \subseteq T(A)$. By Theorem 2.2, we have $\overline{A p r}_{T}\left(S_{2}\right)=S_{1}$. Now by Theorem 2.2 and Theorem 3.6, we get

$$
\begin{aligned}
& \underline{A p r}_{T}(A) S_{1} S_{1} \cap S_{1} \underline{A p r}_{T}(A) S_{1} \cap S_{1} S_{1} \underline{A p r}_{T}(A) \\
& =\underline{A p r}_{T}(A) \underline{A p r} \underline{T}_{T}\left(S_{2}\right) \underline{A p r}_{T}\left(S_{2}\right) \cap \underline{A p r}_{T}\left(S_{2}\right) \underline{A p r}_{T}(A) \underline{A p r}_{T}\left(S_{2}\right) \\
& \cap \underline{A p r}_{T}\left(S_{2}\right) \underline{A p r} \underline{T}_{T}\left(S_{2}\right) \underline{A p r}_{T}(A) \\
& \subseteq \operatorname{Apr}_{T}\left(A S_{2} S_{2}\right) \cap \underline{A p r}_{T}\left(S_{2} A S_{2}\right) \cap \underline{A p r}_{T}\left(S_{2} S_{2} A\right) \\
& =\operatorname{Apr}_{T}\left(A S_{2} S_{2} \cap S_{2} A S_{2} \cap S_{2} S_{2} A\right) \\
& \subseteq \underline{A p r}_{T}(A) \text {. }
\end{aligned}
$$

Similarly we can prove that

$$
\underline{A p r}_{T}(A) S_{1} S_{1} \cap S_{1} S_{1} \underline{A p r}_{T}(A) S_{1} S_{1} \cap S_{1} S_{1} \underline{A p r}_{T}(A) \subseteq \overline{A p r}_{T}(A) .
$$

Thus we obtain that $\underline{A p r}_{T}(A)$ is, if it is non-empty, a quasi-ideal of $S_{1}$.

\section{T-Rough Ideals in the Quotient Ternary Semigroups}

Suppose $S_{1} / T=\left\{T(x): x \in S_{1}\right\}$. It is clear that $S_{1} / T$ is a ternary semigroup.

Definition 4.1. Let $S_{1}$ and $S_{2}$ be two ternary semigroups and $B \subseteq S_{2}$. Let $T: S_{1} \rightarrow \wp^{*}\left(S_{2}\right)$ be a set-valued mapping where $\wp^{*}\left(S_{2}\right)$ denotes the set of all non-empty subsets of $S_{2}$. The generalized lower and upper approximations of $A$ in the quotient ternary semigroups are defined by

$$
\begin{aligned}
& \underline{\underline{A p r}}_{T}(A)=\left\{T(x) \in S_{1} / T: T(x) \subseteq A\right\} \\
& \overline{\overline{A p r}}_{T}(A)=\left\{T(x) \in S_{1} / T: T(x) \cap A \neq \emptyset\right\} .
\end{aligned}
$$

Theorem 4.2. Let $S_{1}$ and $S_{2}$ be two ternary semigroups and $T: S_{1} \rightarrow$ $P\left(S_{2}\right)$ be a set-valued homomorphism. If $A$ is a ternary subsemigroup of $S_{2}$, then

(1) $\overline{\overline{A p r}}_{\rho}(A)$ is a ternary subsemigroup of $S_{1} / T$.

(2) if $T$ is strong, then $\underline{\underline{A p r}}_{\rho}(A)$ is, if it is non-empty, a ternary subsemigroup of $S_{1} / T$.

Proof. (1) Let $T(a), T(b), T(c) \in \overline{\overline{A p r}}_{\rho}(A)$. Then $T(a) \cap A \neq \emptyset, T(b) \cap A \neq \emptyset$ and $T(c) \cap A \neq \emptyset$. So there exist $x \in T(a) \cap A, y \in T(b) \cap A$ and $z \in T(c) \cap A$. 
Since $A$ is a ternary subsemigroup of $S_{2}$, by Theorem $3.9(1), \overline{A p r}_{T}(A)$ is a ternary subsemigroup of $S_{1}$. We have $x y z \in A$ and

$$
x y z \in T(a) T(b) T(c) \subseteq T(a b c) .
$$

Thus $T(a b c) \cap A \neq \emptyset$, which implies that $T(a b c) \subseteq \overline{\overline{A p r}}_{T}(A)$. Hence $\overline{\overline{A p r}}_{T}(A)$ is a ternary subsemigroup of $S_{1} / T$.

(2) Let $T(a), T(b), T(c) \in \underline{\underline{A p r}}_{\rho}(A)$. Then $T(a) \subseteq A, T(b) \subseteq A$ and $T(c) \subseteq$ $A$. Since $A$ is a subsemigroup of $S_{2}$, by Theorem 3.10(1) $\underline{A p r}_{T}(A)$ is a ternary subsemigroup of $S_{1}$, we have

$$
T(a) T(b) T(c)=T(a b c) \subseteq A A A \subseteq A .
$$

This means that $\underline{\underline{A p r}}_{\rho}(A)$ is a ternary subsemigroup of $S_{1} / T$.

Theorem 4.3. Let $S_{1}$ and $S_{2}$ be two ternary semigroups and $T: S_{1} \rightarrow$ $P\left(S_{2}\right)$ be a set-valued homomorphism. If $A$ is a left (right, lateral) ideal of $S_{2}$, then

(1) $\overline{\overline{A p r}}_{T}(A)$ is a left (right, lateral) ideal of $S_{1} / T$.

(2) if $T$ is strong, then $\underline{\underline{\operatorname{Apr}}} \rho(A)$ is, if it is non-empty, a left (right, lateral) ideal of $S_{1} / T$.

Proof. (1) Let $A$ be a left ideal of $S_{2}$. Let $T(x) \in \overline{\overline{A p r}}_{\rho}(A)$ and $T(y), T(z) \in$ $S_{1} / T$. Then $T(x) \cap A \neq \emptyset$, hence $x \in \overline{A p r}_{T}(A)$. Since $A$ is a left ideal of $S_{2}$, by Theorem 3.9(2), $\overline{A p r}_{T}(A)$ is a left ideal of $S_{1}$. So, we have

$$
y z x \in \overline{\operatorname{Apr}}_{\rho}(A) .
$$

Now, for every $m=y z x$, we have $T(m) \cap A \neq \emptyset$. On the other hand, from $m=$ $y z x$, we obtain $T(m) \subseteq T(y) T(z) T(x)$. Therefore $T(y) T(z) T(x) \subseteq \overline{\overline{A p r}}_{\rho}(A)$. This means that $\overline{\overline{A p r}}_{\rho}(A)$ is a left ideal of $S_{1} / T$.

(2) Let $A$ be a left ideal of $S_{2}$. Let $T(x) \in \overline{\overline{A p r}}_{\rho}(A)$ and $T(y), T(z) \in S_{1} / T$. Then, $T(x) \subseteq A$, which implies $x \in \operatorname{Apr}_{T}(A)$. Since $A$ is a left ideal of $S_{2}$, by Theorem 3.10(2), $\underline{A p r}_{T}(A)$ is a left ideal of $S_{1} / T$. Thus, we have

$$
y z x \in \underline{\operatorname{Apr}}_{\rho}(A) .
$$

Now, for every $m=y z x$, we have $m \in \underline{A p r} \rho \rho$, which implies that $T(m) \subseteq A$. Hence, $T(m) \in \underline{\underline{\operatorname{Apr}}} \rho(A)$. On the other hand, from $m=y z x$, we have $T(m) \subseteq$ 
$T(z) T(y) T(x)$. Therefore $T(z) T(y) T(x) \subseteq \underline{\underline{A p r}} \rho(A)$. This means that $\underline{\underline{\text { Apr }}} \rho \rho(A)$ is, if it is non-empty, a left ideal of $S_{1} / T$. The other cases can be seen in a similar way.

Theorem 4.4. Let $S_{1}$ and $S_{2}$ be two ternary semigroups and $T: S_{1} \rightarrow$ $P\left(S_{2}\right)$ be a set-valued homomorphism. If $A$ is a bi-ideal of $S_{2}$, then

(1) $\overline{\overline{A p r}}_{T}(A)$ is a bi-ideal of $S_{1} / T$.

(2) if $T$ is strong, then $\underline{\underline{A p r}} \rho \rho(A)$ is, if it is non-empty, a bi-ideal of $S_{1} / T$.

Proof. The proof is similar to the proof of the Theorem 4.3.

Theorem 4.5. Let $S_{1}$ and $S_{2}$ be two ternary semigroups and $T: S_{1} \rightarrow$ $P\left(S_{2}\right)$ be a set-valued homomorphism. If $A$ is an interior ideal of $S_{2}$, then

(1) $\overline{\overline{A p r}}_{T}(A)$ is an interior ideal of $S_{1} / T$.

(2) if $T$ is strong, then $\underline{\underline{A p r}} \rho(A)$ is, if it is non-empty, an interior ideal of $S_{1} / T$.

Proof. The proof is similar to the proof of the Theorem 4.3.

\section{References}

[1] Z. Pawlak, Rough sets, Int. J. Comput. Inform. Sci., 11 (1982), 341-356.

[2] R. Biswas, S. Nanda, Rough groups and rough subgroups, Bull. Polish Acad. Sci. Math., 42 (1994), 251-254.

[3] Q.M. Xiao, Z.L. Zhang, Rough prime ideals and rough fuzzy prime ideals in semigroups, Inform. Sci., 176 (2006), 725-733.

[4] P. Petchkhaew, R. Chinram, Fuzzy, rough and rough fuzzy ideals in ternary semigroups, Int. J. Pure Appl. Math., 56 (2009), 21-36.

[5] N. Kuroki, Rough ideals in semigroups, Inform. Sci., 100 (1997), 139-163.

[6] M. Shabir, N. Rehman, Roughness in ternary semigroups, Quasigroups Related Systems, 19 (2011), 101-108.

[7] M. Aslam, S. Abdullah, B. Davvaz, N. Yaqoob, On rough M-hypersystems and fuzzy M-hypersystems in $\Gamma$-semihypergroups, Neural Comput. Applic., 21, No. 1 (2012), 281-287. 
[8] M. Aslam, M. Shabir, N. Yaqoob, A. Shabir, On rough $(\mathrm{m}, \mathrm{n})$-bi-ideals and generalized rough (m,n)-bi-ideals in semigroups, Ann. Fuzzy Math. Inform., 2, No. 2 (2011), 141-150.

[9] N. Yaqoob, M. Aslam, B. Davvaz, A.B. Saeid, On rough $(\mathrm{m}, \mathrm{n})$ bi- - hyperideals in $\Gamma$-semihypergroups, U.P.B. Scientific Bulletin, Series A, 75, No. 1 (2013), 119-128.

[10] N. Yaqoob, M. Aslam, R. Chinram, Rough prime bi-ideals and rough fuzzy prime bi-ideals in semigroups, Ann. Fuzzy Math. Inform., 3, No. 2 (2012), 203-211.

[11] N. Yaqoob, Applications of rough sets to $\Gamma$-hyperideals in left almost $\Gamma$ semihypergroups, Neural Comput. Applic., 21, No. 1 (2012), 267-273.

[12] N. Yaqoob, M. Aslam, K. Hila, Rough fuzzy hyperideals in ternary semihypergroups, Adv. Fuzzy Syst., 2012 (2012), Article ID 595687, 9 pages.

[13] N. Yaqoob, S. Abdullah, N. Rehman, M. Naeem, Roughness and fuzziness in ordered ternary semigroups, World Appl. Sci. J., 17, No. 12 (2012), 1683-1693.

[14] N. Yaqoob, Rough approximations in left almost groups, Int. J. Algebra Stat., 1, No. 1 (2012) 100-109.

[15] N. Yaqoob, R. Chinram, On prime $(\mathrm{m}, \mathrm{n})$ bi-ideals and rough prime $(\mathrm{m}, \mathrm{n})$ bi-ideals in semigroups, Far East J. Math. Sci., 62, No. 2 (2012), 145-159.

[16] N. Yaqoob, M. Aslam, Yao's generalized rough approximations in left almost semigroups, Ann. Fuzzy Math. Inform., 3, No. 1 (2012), 119-131.

[17] B. Davvaz, Roughness in rings, Inform. Sci., 164 (2004), 147-163.

[18] B. Davvaz, Approximations in hyperrings, J. Mult.-Valued Logic Soft Comput., 15 (2009) 471-488.

[19] B. Davvaz, Rough subpolygroups in a factor polygroup, J. Intell. Fuzzy Syst., 17, No. 6 (2006) 613-621.

[20] O. Kazancı, S. Yamak, B. Davvaz, The lower and upper approximations in a quotient hypermodule with respect to fuzzy sets, Inform. Sci., 178 (2008) 2349-2359. 
[21] V. Leoreanu-Fotea, The lower and upper approximations in a hypergroup, Inform. Sci., 178 (2008) 3605-3615.

[22] B. Davvaz, A short note on algebraic T-rough sets, Inform. Sci., 178 (2008), 3247-3252.

[23] Q.M. Xiao, T-roughness in semigroups, IEEE Trns. Comput. Sci. Aut. Eng., 2 (2011), 391-394.

[24] S.B. Hosseini, N. Jafarzadeh, A. Gholami, T-rough ideal and T-rough fuzzy ideal in a semigroup, Adv. Materials Res., 433 (2012), 4915-4919.

[25] S.B. Hosseini, M. Saberifar, Some properties of a set-valued homomorphism on modules, J. Basic. Appl. Sci. Res., 2, No. 5 (2012), 5426-5430. 